\title{
A Monte Carlo Algorithm for State and Parameter Estimation of Extended Targets ${ }^{\star}$
}

\author{
Donka Angelova $^{1}$ and Lyudmila Mihaylova ${ }^{2}$ \\ ${ }^{1}$ Institute for Parallel Processing, Bulgarian Academy of Sciences \\ 25A Acad. G. Bonchev St, 1113 Sofia, Bulgaria \\ donka@bas.bg \\ ${ }^{2}$ Department of Communication Systems, Lancaster University, \\ South Drive, Lancaster LA1 4WA, UK \\ mila.mihaylova@ieee.org
}

\begin{abstract}
This paper considers the joint state and parameter estimation of extended targets. Both the target kinematic states, position and speed, are estimated with the target extent parameters. The developed algorithm is applied to a ship, whose shape is modelled by an ellipse. A Bayesian sampling algorithm with finite mixtures is proposed for the evaluation of the extent parameters whereas a suboptimal Bayesian interacting multiple model (IMM) filter estimates the kinematic parameters of the maneuvering ship. The algorithm performance is evaluated by Monte Carlo comparison with a particle filtering approach.
\end{abstract}

\section{Introduction}

The increasing interest in simulation-based Bayesian methods for analysis of dynamic models has been resulted in a variety of powerful techniques for filtering and prediction of complex dynamic systems [1,2]. The problem of state and parameter estimation of dynamic systems has many applications such as in signal processing, machine learning, robotics, target (e.g., aircraft, ship) tracking [3, 4]. In the present work, a Monte Carlo (MC) algorithm is applied to ship size evaluation in the framework of state filtering (tracking) of a maneuvering ship, modeled by a Markovian jump system.

Most of the target tracking algorithms available in the literature consider the moving object as a single point and estimate its state vector based on the incoming sensor data, e.g. range and bearing. However, recent sensor systems are able to resolve individual features or measurement sources on an extended object. The possibility to additionally make use of this measurements is referred to extended target tracking. For example, a high-resolution radar can provide a measure of down-range object extent given a reasonable signal-to-noise ratio [5,6]. This valuable information can help for more precise estimation of the object behaviour. It can assist in resolving measurement association uncertainty in situations of closely spaced objects in dense clutter. Furthermore, the knowledge of objects size is especially important for the purposes of classification.

\footnotetext{
${ }^{\star}$ Research supported in part by the Bulgarian Foundation for Scientific Investigations: I-1202/02,I-1205/02,MI-1506/05 and by Center of Excellence BIS21++, 016639.
} 
In this paper we address the problem of extended target tracking. There exist several ways for modelling object extent parameters. Models, based on measurements of individual points on object require complicated techniques for multiple hypotheses testing [7]. A simple ellipsoidal object model is proposed in [5] and adopted in our work. The lengths of the major and minor axes of the ellipse have to be calculated, based on the measurements of down-range extent. Shape parameters are included in [5] in the state vector together with kinematic parameters and are estimated by Extended and Unscented Kalman Filters (EKFs and UKFs) and particle filtering. However, it is pointed out is [5] that the EKF implementation is prone to divergence due to high nonlinearity conditions and a straightforward particle filter can avoid this problem.

Having in mind the inferences in [5,6], we develop a Bayesian algorithm able to deal with the nonlinear estimation problem. Taking into account the possibility for subsequent classification, we assume that the unknown shape parameters are defined over the discrete set of values, with a given prior distribution. Within this formulation of the problem, data augmentation (DA) algorithm for finite mixture estimation [9] offers an alternative solution. DA represents a special case of Gibbs sampling [2] and belongs to the class of Markov Chain Monte Carlo (MCMC) methods. We develop a DA procedure for the parameter estimation, along with an IMM algorithm for kinematic state estimation. The scheme implemented here is inspired by the ideas in [8].

The paper is organised as follows. Section 2 describes the system dynamics and measurement model. Section 3 presents the formulation of the problem. The designed DA algorithm is presented in Section 4 . Section 5 illustrates and compares the performance of the proposed algorithm with a particle filter (PF). Conclusions are given in Section 6

\section{System Dynamics and Measurement Models}

Target motion tracking is performed by a recursive reconstruction of the state probability density function given the available prior information and current measurement data. Prior information includes dynamic models, models of the measurement process, initial state and noise probability distributions.

System Model. Consider the following model of a discrete-time jump Markov system, describing object dynamics and sensor measurements

$$
\begin{aligned}
\boldsymbol{x}_{k} & =\boldsymbol{f}\left(m_{k}, \boldsymbol{x}_{k-1}, \boldsymbol{\theta}\right)+\boldsymbol{g}\left(m_{k}, \boldsymbol{\theta}\right) \boldsymbol{w}_{k}, \\
\boldsymbol{z}_{k} & =\boldsymbol{h}\left(m_{k}, \boldsymbol{x}_{k}, \boldsymbol{\theta}\right)+\boldsymbol{d}\left(m_{k}, \boldsymbol{\theta}\right) \boldsymbol{v}_{k}, k=1,2, \ldots,
\end{aligned}
$$

where $\boldsymbol{x}_{k} \in \mathbb{R}^{n_{x}}$ is the base (continuous) state vector, with transition function $\boldsymbol{f}$, $\boldsymbol{z}_{k} \in \mathbb{R}^{n_{z}}$ is the measurement vector with measurement function $\boldsymbol{h}$, and $\boldsymbol{\theta} \in \boldsymbol{\Theta}$ is a vector, containing unknown static parameters. The noises $\boldsymbol{w}_{k}$ and $\boldsymbol{v}_{k}$ are independent identically distributed (i.i.d.) Gaussian processes having characteristics $\boldsymbol{w}_{k} \sim N(\mathbf{0}, \boldsymbol{Q})$ and $\boldsymbol{v}_{k} \sim N(\mathbf{0}, \boldsymbol{R})$, respectively. All vectors and matrices are assumed of appropriate dimensions. The modal (discrete) state $m_{k} \in \mathbb{S} \triangleq\{1,2, \ldots, s\}$ is a first-order Markov chain with transition probabilities $p_{i j} \triangleq \operatorname{Pr}\left\{m_{k}=j \mid m_{k-1}=i\right\},(i, j \in \mathbb{S})$ and initial probability distribution $P_{0}(i) \triangleq \operatorname{Pr}\left\{m_{0}=i\right\}, i \in \mathbb{S}$, such that $P_{0}(i) \geq 0$, and $\sum_{i=1}^{s} P_{0}(i)=1 . k=1,2, \ldots$ is a discrete time. 
Consider a base state vector in the form $\boldsymbol{x}_{k}=\left(x_{k}, \dot{x}_{k}, y_{k}, \dot{y}_{k}\right)^{\prime}$, where $x$ and $y$ specify the ship position with respect to an observer position, assumed known, and $(\dot{x}, \dot{y})$ is the velocity in the Cartesian plane, centered at the observer location. All possible $s$ motion regimes of the maneuvering ship are modelled by the modal state variable $m$. The static parameter vector $\boldsymbol{\theta}=(\ell, \gamma)^{\prime}$ contains shape parameters: the length of the major axis of the ship ellipse $\ell$ and the ratio of the lengths of the minor and major axes $\gamma$.

Measurement Equation. Similarly to [5,6], we assume, that a high-resolution radar provides measurements of range $r$ and bearing $\beta$ to the object centroid, as well as the object down-range extent $L$ along the observer-object line-of-sight (LOS). The relationship between $L$ and the angle $\phi$ between the major axis of the ellipse and the targetobserver LOS is given by

$$
L(\phi)=\ell \sqrt{\cos ^{2} \phi+\gamma^{2} \sin ^{2}(\phi)} .
$$

The measurement function $\boldsymbol{h}$ in (2) is nonlinear,

$$
\boldsymbol{h}\left(\boldsymbol{x}_{k}, \boldsymbol{\theta}\right)=\left(\begin{array}{c}
\sqrt{\left(x_{k}-x_{o}\right)^{2}+\left(y_{k}-y_{o}\right)^{2}} \\
\arctan \left(\left(y_{k}-y_{o}\right) /\left(x_{k}-x_{o}\right)\right) \\
L\left(\phi\left(\boldsymbol{x}_{k}\right)\right)
\end{array}\right)
$$

where the measurement vector is $\boldsymbol{z}_{k}=\left(r_{k}, \beta_{k}, L_{k}\right)^{\prime}$. Here $\left(x_{o}, y_{o}\right)$ is the location of the observer. If it is assumed that the target ellipse is oriented so that its major axis is parallel to the velocity vector $(\dot{x}, \dot{y})$ then from (3) the along-range target extent can be written in terms of the state vector and $\boldsymbol{\theta}$ as

$$
L\left(\phi\left(\boldsymbol{x}_{k}\right)\right)=\boldsymbol{\theta}(1) \sqrt{\cos ^{2} \phi\left(\boldsymbol{x}_{k}\right)+\boldsymbol{\theta}(2)^{2} \sin ^{2} \phi\left(\boldsymbol{x}_{k}\right)}
$$

where $\phi\left(\boldsymbol{x}_{k}\right)=\arctan \left(\left(x_{k} \dot{y}_{k}-\dot{x}_{k} y_{k}\right) /\left(x_{k} \dot{x}_{k}+y_{k} \dot{y}_{k}\right)\right)$.

The problem that we consider has own particularities: the measurements of $L$ are not used for the base state vector estimation. The kinematic states are estimated through $r$ and $\beta$. The estimated kinematic states are, however, used for the estimation of $\ell$ and $\gamma$. This is the motivation for applying separate estimators to the two estimation problems.

\section{Problem Formulation}

The goal is to estimate the state vector $\boldsymbol{x}_{k}$ and the extent parameter vector $\boldsymbol{\theta}$, based on all available measurement information $\boldsymbol{Z}^{k}=\left\{\boldsymbol{z}_{1}, \boldsymbol{z}_{2}, \ldots, \boldsymbol{z}_{k}\right\}$. If we can calculate the posterior joint state-size probability density function (PDF)

$$
p\left(\boldsymbol{x}_{k}, \boldsymbol{\theta} \mid \boldsymbol{Z}^{k}\right)=p\left(\boldsymbol{\theta} \mid \boldsymbol{x}_{k}, \boldsymbol{Z}^{k}\right) p\left(\boldsymbol{x}_{k} \mid \boldsymbol{Z}^{k}\right)
$$

then for any integrable function $\hbar\left(\boldsymbol{x}_{k}, \boldsymbol{\theta}\right)$ the required estimate is given by

$$
E\left\{\hbar\left(\boldsymbol{x}_{k}, \boldsymbol{\theta}\right) \mid \boldsymbol{Z}^{k}\right\}=\iint \hbar\left(\boldsymbol{x}_{k}, \boldsymbol{\theta}\right) p\left(\boldsymbol{\theta} \mid \boldsymbol{x}_{k}, \boldsymbol{Z}^{k}\right) p\left(\boldsymbol{x}_{k} \mid \boldsymbol{Z}^{k}\right) d \boldsymbol{\theta} d \boldsymbol{x}_{k} .
$$


If we denote the $l$-th mode history, realised by a Markovian jump system through time $k$ as $m_{k}^{l}, l=1, \ldots, \varepsilon_{k}$ then the conditional PDF of the state is obtained as a Gaussian mixture with an exponentially increasing number of terms [10]

$$
p\left(\boldsymbol{x}_{k} \mid \boldsymbol{Z}^{k}\right)=\sum_{l=1}^{\varepsilon_{k}} p\left(\boldsymbol{x}_{k} \mid m_{k}^{l}, \boldsymbol{Z}^{k}\right) P\left(m_{k}^{l} \mid \boldsymbol{Z}^{k}\right) .
$$

The exponentially increasing computations can be avoided by different ways of combining histories of models. For example, the Interacting Multiple Model (IMM) filter [10] provides an approximate state estimate $\hat{\boldsymbol{x}}_{k}=E\left\{\hbar\left(\boldsymbol{x}_{k}\right) \mid \boldsymbol{Z}^{k}\right\}$ and its associated covariance matrix $\boldsymbol{P}_{k}$, by using $s$ working in parallel EKFs. Then the estimate of $\boldsymbol{\theta}$ can be expressed as $\hat{\boldsymbol{\theta}}_{k}=E\left\{\hbar\left(\boldsymbol{\theta}_{k}\right) \mid \hat{\boldsymbol{x}}_{k}, \boldsymbol{Z}^{k}\right\}$. Note that the $k$ index of $\boldsymbol{\theta}$ indicates that it is calculated based on the information up to time instant $k$, not that $\boldsymbol{\theta}$ is time-varying.

$\mathrm{MC}$ algorithms (PF or MCMC) can be applied to the highly nonlinear extent estimation problem. The use PF for the first step is not justifiable, as ships exhibit moderate maneuvers and the time interval between the measurements is rather short. Since the size estimate could be obtained along with the filtering process, but not necessary online, the more precise MCMC algorithm can be applied.

The scheme implemented here is similar to [8]. It comprises the following steps. On receipt of a new measurement $\boldsymbol{z}_{k}$ :

a) run the IMM algorithm with the previous state estimate $\hat{\boldsymbol{x}}_{k-1}$ in order to update the current state estimate $\hat{\boldsymbol{x}}_{k}$.

b) find the estimate $\hat{\boldsymbol{\theta}}_{k}$ of the parameter vector $\boldsymbol{\theta}$ based on the previous estimates $\hat{\boldsymbol{\theta}}_{k-1}, \hat{\boldsymbol{x}}_{k}$, and the measurement likelihood $p\left(\boldsymbol{z}_{k} \mid \hat{\boldsymbol{\theta}}_{k-1}, \boldsymbol{z}_{k-1}\right)$, by PF or DA scheme.

\section{Extent Parameters Estimation by Stochastic Simulation}

Based on a priori information about ship types, we assume that $\boldsymbol{\theta}$ takes values from a known discrete set $\boldsymbol{\theta} \in \mathbb{T} \triangleq\{1,2, \ldots, t\}$ with known prior distribution: $P_{\boldsymbol{\theta}_{0}}(i) \triangleq$ $\operatorname{Pr}\{\boldsymbol{\theta}=i\}, i \in T$, such that $P_{\boldsymbol{\theta}_{0}}(i) \geq 0$, and $\sum_{i=1}^{t} P_{\boldsymbol{\theta}_{0}}(i)=1$. Let us suppose that along-range extent measurements $L_{k}, k=1, \ldots, n, \ldots$ have Gaussian distributed zeromean errors with known variance $R_{L}$. The PDF of the measurement $L_{k}$ is represented in the following mixture form [9, 11]:

$$
p\left(L_{k} \mid \boldsymbol{\pi}, \boldsymbol{\theta}, \boldsymbol{x}_{k}\right)=\sum_{j=1}^{t} \pi_{j} G\left(L_{k} \mid \boldsymbol{\theta}_{j}, \boldsymbol{x}_{k}\right),
$$

where $\boldsymbol{\pi}=\left(\pi_{1}, \ldots, \pi_{t}\right)$ are the mixture proportions which are constrained to be nonnegative and sum to unity. $G\left(L_{k} \mid \boldsymbol{\theta}_{j}, \boldsymbol{x}_{k}\right) \sim N\left(L_{k} ; L\left(\boldsymbol{\theta}_{j}, \hat{\boldsymbol{x}}_{k}\right), R_{L}\right)$ is a Gaussian density and $L\left(\boldsymbol{\theta}_{j}, \hat{\boldsymbol{x}}_{k}\right)$ is the measurement prediction, calculated according to (5). Thus, the task is reduced to the well known finite mixture estimation problem: for the mixture model (9) with known component PDFs $G\left(L_{k} \mid \boldsymbol{\theta}_{j}, \boldsymbol{x}_{k}\right)$, one needs to estimate the unknown weights $\pi=\left(\pi_{1}, \ldots, \pi_{t}\right)$, given a sequence of independent observations $L_{1}, L_{2}, \ldots, L_{n}$. The mixture component with a maximum weight identifies the 
most probable ship type. The estimate of the extent parameters can be calculated as the weighted by $\pi$ sum of the possible $\boldsymbol{\theta}$ values in the set.

Mixture Weights Estimation by Data Augmentation. DA algorithm approximately evaluates the mixture posterior distribution, relying on the missing data structure of mixture model. Generally, the mixture model is given by the observation of $n$ independent random variables $y_{1}, \ldots, y_{n}$ from a $t$-component mixture [9, 11],

$$
\digamma\left(y_{k}\right)=\sum_{j=1}^{t} \pi_{j} \digamma_{j}\left(y_{k}\right), k=1, \ldots, n,
$$

where the densities $\digamma_{j}, j=1, \ldots, t$ are known or are known up to a parameter and the proportions $\pi_{j}$ satisfy the above conditions. We consider the special case, where only the weights $\pi$ have to be estimated. According to [9], the mixture model can always be expressed in terms of missing (or incomplete) data. That is, define vectors $\delta(k)=\left(\delta_{1}(k), \delta_{2}(k), \ldots, \delta_{t}(k)\right), k=1,2, \ldots, n$ with components $\delta_{j}(k) \in$ $\{0,1\}, j=1,2, \ldots, t$, which indicate that the measurement $y_{k}$ has density $\digamma_{j}\left(y_{k}\right)$ [8]. The model is hierarchical with, on top, the true parameters of the mixture, $\pi$, then the missing data whose distribution depends on $\boldsymbol{\pi}, \boldsymbol{\delta} \sim p(\boldsymbol{\delta} \mid \boldsymbol{\pi})$, and, at the bottom, the observed data $\boldsymbol{y} \sim p(\boldsymbol{y} \mid \boldsymbol{\pi}, \boldsymbol{\delta})$. Starting with an initial value $\boldsymbol{\pi}^{(0)}$, the algorithm implements two-step iterative scheme: at the iteration $u, u=1,2, \ldots$

a) generate $\boldsymbol{\delta}^{(u)} \sim p\left(\boldsymbol{\delta} \mid \boldsymbol{y}, \boldsymbol{\pi}^{(u)}\right)$ from a multinomial distribution with weights proportional to the observation likelihoods: $\delta_{j}^{(u)}(k) \propto \pi_{j}^{(u)} \digamma_{j}\left(y_{k}\right)$;

b) generate $\boldsymbol{\pi}^{(u+1)} \sim p\left(\boldsymbol{\pi} \mid \boldsymbol{y}, \boldsymbol{\delta}^{(u)}\right)$.

Since the conjugate priors on $\pi$ are with Dirichlet distributions (DD) $\mathcal{D}\left(\alpha_{1}, \ldots, \alpha_{t}\right)$ [9], $\boldsymbol{\pi}^{(u+1)}$ is generated according to the DDs with parameters, depending on the missing data. Bayesian sampling produces an ergodic Markov chain $\left(\boldsymbol{\pi}^{(u)}\right)$ with stationary distribution $p(\boldsymbol{\pi} \mid \boldsymbol{y})$. Thus, after $u_{0}$ initial (warming up) steps, a set of $U$ samples $\boldsymbol{\pi}^{\left(u_{0}+1\right)}, \ldots, \boldsymbol{\pi}^{\left(u_{0}+U\right)}$ are approximately distributed as $p(\boldsymbol{\pi} \mid \boldsymbol{y})$ and, due to ergodicity, averaging can be made with respect to time [8,9].

In the next scheme, the sequence of observations $y_{k}, k=1, \ldots$ is replaced by the along-range extent measurements $L_{k}, k=1, \ldots$. The joint IMM and data augmentation scheme is given below. DA is realised in a sliding window mode.

\section{Algorithm Outline}

For $k=1,2, \ldots$

- Run the IMM algorithm with the previous state vector $\hat{\boldsymbol{x}}_{k-1}$, covariance matrix $\boldsymbol{P}_{k-1}$ and posterior model probability vector $\boldsymbol{\mu}_{k-1}$ to update the current estimate $\hat{\boldsymbol{x}}_{k}$, together with $\boldsymbol{P}_{k}$ and $\boldsymbol{\mu}_{k}$.

- Compute Mixture Components Conditional PDFs

$$
\tilde{G}_{j}(k) \triangleq G\left(L_{k} \mid \boldsymbol{\theta}_{j}, \boldsymbol{x}_{k}\right) \propto \exp \left[-0.5\left(L_{k}-L\left(\boldsymbol{\theta}_{j}, \hat{\boldsymbol{x}}_{k}\right)\right)^{T} R_{L}^{-1}\left(L_{k}-L\left(\boldsymbol{\theta}_{j}, \hat{\boldsymbol{x}}_{k}\right)\right)\right]
$$

- Implement Data Augmentation

$$
\text { - Initialisation: } \pi^{(0)}=\pi(k-1)
$$


- Iterations $\left(u=0,1, \ldots, u_{0}+U-1\right)$

* Missing Data Conditional Probability Mass Functions (PMFs)

$$
q_{j}^{(u)}(l)=\frac{\pi_{j}^{(u)} \tilde{G}_{j}(l)}{\sum_{j=1}^{t} \pi_{j}^{(u)} \tilde{G}_{j}(l)}, \quad l=1,2, \ldots k, \quad j=1,2, \ldots t
$$

* Missing Data Generation (Multinomial Sampling)

$$
\delta^{(u)}(l)=(0, \ldots, 0,1,0, \ldots, 0) \sim\left\{q_{j}^{(u)}(l)\right\}_{j=1}^{t}, \quad l=1,2, \ldots k
$$

* Parameter Evaluation (Dirichlet Distribution Sampling)

$$
\pi^{(u+1)} \sim \mathcal{D}\left(\pi ; \alpha_{1}+\sum_{l=1}^{k} \delta_{1}^{(u)}(l), \ldots, \alpha_{t}+\sum_{l=1}^{k} \delta_{t}^{(u)}(l)\right)
$$

- Calculate the Output Estimates

$$
\pi(k)=\frac{1}{U} \sum_{\sigma=1}^{U} \pi^{\left(u_{0}+\sigma\right)} \quad \text { and } \quad \hat{\boldsymbol{\theta}}_{k}=\sum_{j=1}^{t} \pi_{j}(k) \boldsymbol{\theta}_{j}
$$

\section{Simulation Results}

The algorithm performance is evaluated by simulations over trajectories, including consecutive segments of uniform motion and maneuvers (a typical scenario is shown in Fig 1 (a)). The observer is static, located at the origin of $x-y$ plane. The initial target state is $\boldsymbol{x}_{0}=(18 e 3,-14,90 e 3,5)^{\prime}$. It performs two turn maneuvers with a normal acceleration of $\pm 1.4\left[\mathrm{~m} / \mathrm{s}^{2}\right]$. The object length is $\ell=50[\mathrm{~m}]$ and the ratio of the lengths of the minor and major axes (aspect ratio) is $\gamma=0.2$. The sensor parameters are as follows [6]: sampling interval $T=0.2[s]$; the standard deviations of measurement errors along range, azimuth and along-range target extent are respectively:

$$
\sigma_{r}=5[\mathrm{~m}], \sigma_{\beta}=0.2 \mathrm{deg} \text { and } \sigma_{L}=5[\mathrm{~m}] .
$$

Root-Mean Squared Errors (RMSEs) [10] are selected as a quantitative measure for the algorithm performance evaluation.

An IMM algorithm with $s=3$ models is designed. The first model corresponds to the nearly constant velocity motion. The next two models are matched to the nearly coordinated turn maneuvers with a turn rate of $\omega= \pm 1.4[\mathrm{rad} / \mathrm{s}]$. The form of the transition matrices in (1) for these models can be found in [10].

A particle filter for extent parameters estimation is realised for the purposes of comparison with DA algorithm, with $N=300$ particles. Similarly to the procedure, described in [1] (Ch.10), $N$ particles $\left(\boldsymbol{\theta}^{(i)}\right)_{i=1}^{N}$ are generated according to a priori normal distribution with mean, corresponding to the true $\boldsymbol{\theta}$. After that the particles are predicted according to a normal distribution with small deviations to provide "artificial 

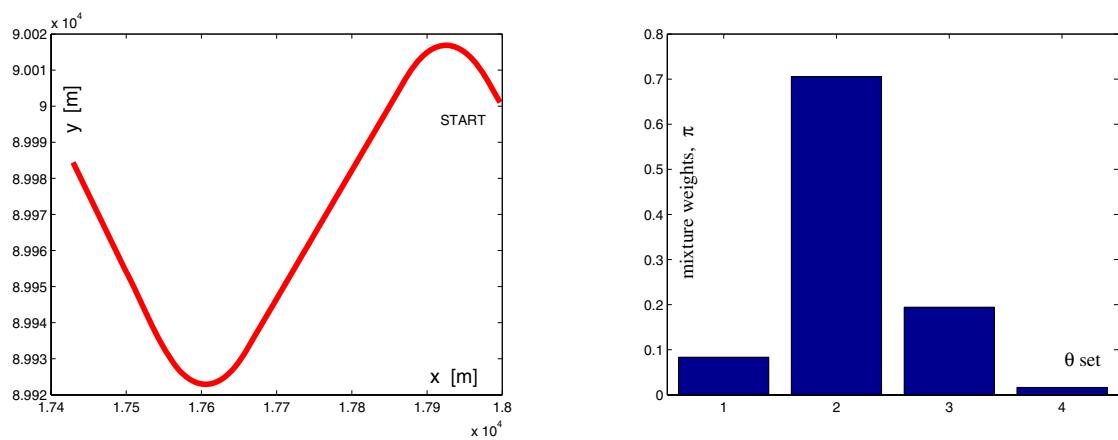

Fig. 1. Object trajectory and Mixture proportions $\pi$
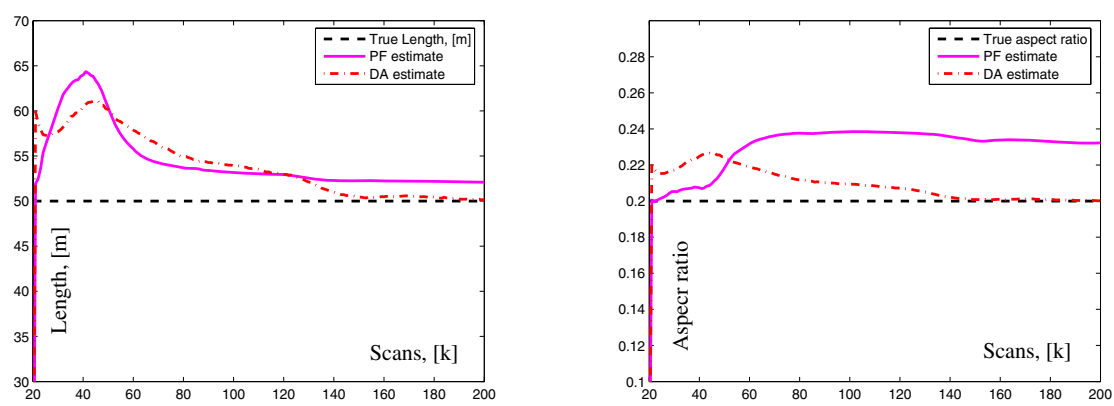

Fig. 2. PF and DA comparison: True and estimated $\ell$ and $\gamma$
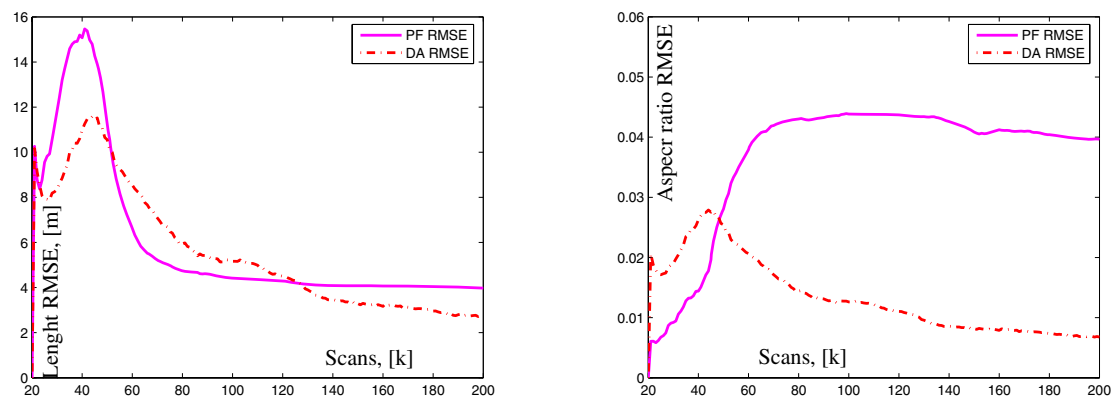

Fig. 3. PF and DA comparison: RMSE of $\ell$ and $\gamma$

evolution" of parameters. Then the particle weights are evaluated using likelihoods of the received measurements and finally resampling is implemented according to a known rule.

In accordance with (Sec. 4), we assume that $\boldsymbol{\theta}$ takes values from a discrete set of $t=4$ components: $\{(30,0.15),(50,0.2),(70,0.25),(100,0.3)\}$ with equal initial probabilities. $\boldsymbol{\theta}_{2}$ corresponds to the true $\boldsymbol{\theta}$. The mixture proportions $\pi_{j}, j=1, \ldots, t$, estimated by the DA procedure are given in Fig 1 b). It can be seen that DA identifies 
the correct $\boldsymbol{\theta}$ with a high probability. The results are obtained from one MC realisation and confirm the reliability of the algorithm for classification tasks.

Figs. (2) and (3) demonstrate the better performance of the DA in comparison with the PF: the estimation errors of $\ell$ and $\gamma$ obtained by the PF are quite large. It is natural to expect a better performance of DA scheme. It has additional prior information, available from the $\boldsymbol{\theta}$ set. DA is an off-line procedure and processes the cumulative measurement information. In addition, PF involves additional noise, necessary for prediction which deteriorates the estimation accuracy of the parameters (they are fixed). Its performance is highly sensitive to the choice of noise deviations, which are design parameters. However, the better performance is achieved at the increased computational time. The relative execution time is approximately 17:1 in PF favour.

\section{Conclusion}

An alternative solution to the problem of extended object tracking is proposed in this paper. Two different Bayesian algorithms are developed for kinematic state and size parameter estimation of a ship, based on positional and along-range object extent measurements. A Monte Carlo algorithm (Data augmentation) is designed for length and aspect ratio assessment of the elliptical ship shape. The performance of the proposed algorithm is evaluated by Monte Carlo simulation. The results show that DA is capable to deal with highly nonlinear relationships between states, parameters, measurements and complicated target-observer geometry. A combined IMM-DA procedure is proposed which provides accurate joint state-parameter estimation and reliable identification of the ship type.

\section{References}

1. Doucet, A., de Freitas, N., Gordon, N. (ed.): Sequential Monte Carlo Methods in Practice. Springer-Verlag, New York, (2001).

2. Liu, J.: Monte Carlo Strategies in Scientific Computing. Springer-Verlag, New York, (2003).

3. Storvik, G.: Particle filters in state space models with the presence of unknown static parameters. IEEE. Trans. of Signal Processing, Vol. 50, No. 2, (2002) 281-289.

4. Doucet, A., Tadic, V.: Parameter estimation in general state-space models using particle methods. Ann. Inst. Stat. Math., vol. 55, No. 2, (2003) 409-422.

5. Salmond, D., Parr, M.: Track maintenance using measurements of target extent. IEE Proc.Radar Sonar Navig., Vol. 150, No. 6, (2003) 389-395.

6. Ristic, B., Salmond, D.: A study of a nonlinear filtering problem for tracking an extended target. Proc. Seventh Intl. Conf. on Information Fusion, (2004) 503-509.

7. Vermaak, J., N. Ikoma, S. Godsill: Sequential Monte Carlo Framework for Extended Object Tracking. IEE Proc.-Radar Sonar Navig., Vol. 152, No. 5 (2005), 353-363.

8. Jilkov, V., Li,X. Rong, Angelova, D.: Estimation of Markovian Jump Systems with Unknown Transition Probabilities through Bayesian Sampling. LNCS, Vol. 2542 (2003), SpringerVerlag, Berlin, pp. 307-315.

9. Diebolt, J., Robert, C.P.: Estimation of Finite Mixture Distributions through Bayesian Sampling. J. of Royal Statist. Soc. B 56, No. 4, (1994) 363-375.

10. Bar-Shalom, Y., Li, X.R., Kirubarajan, T.: Estimation with Applications to Tracking and Navigation: Theory, Algorithms, and Software. Wiley, New York (2001).

11. Stephens, M.: Bayesian methods for mixture of normal distributions. PhD Thesis, 1997. 